Research Paper

\title{
The Upregulation of Trophinin-Associated Protein (TROAP) Predicts a Poor Prognosis in Hepatocellular Carcinoma
}

\author{
Hao $\mathrm{Hu}^{1 *}$, Liang $\mathrm{Xu}^{2 *}$, Yan Chen ${ }^{3}$, Shao-Ju Luo', Ying-zi Wu ${ }^{3}$,Shi-Hua $\mathrm{Xu}^{1}$, Meng-Ting Liu1, Fen Lin², Yan

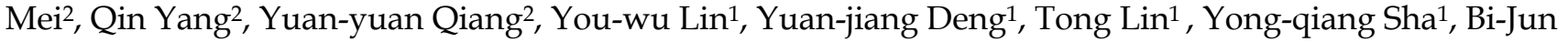 \\ Huang ${ }^{\circledR}$, Shi-Jun Zhang ${ }^{\bowtie}$ \\ 1. Department of Chinese Medicine, the First Affiliated Hospital, Sun Yat-sen University, Guangzhou 510080, Guangdong, P. R. China \\ 2. State Key Laboratory of Oncology in South China; Collaborative Innovation Center for Cancer Medicine, Sun Yat-Sen University Cancer Center, Guangzhou \\ 510060, P. R. China \\ 3. Department of Chinese Medicine, the Third Affiliated Hospital, Sun Yat-sen University, Guangzhou 510630, Guangdong, P. R. China. \\ * The first two authors contributed to the study equally \\ $\square$ Corresponding authors: Bi-Jun Huang, State Key Laboratory of Oncology in South China; Collaborative Innovation Center for Cancer Medicine, Sun Yat-sen \\ University Cancer Center, Guangzhou 510060, P. R. China. Email: huangbj@sysucc.org.cn. Dr. Shi-Jun Zhang, Department of Chinese Medicine, the First \\ Affiliated Hospital, Sun Yat-sen University, Guangzhou 510080, Guangdong, P. R. China; Email: zhsjun1967@hotmail.com. \\ (c) Ivyspring International Publisher. This is an open access article distributed under the terms of the Creative Commons Attribution (CC BY-NC) license \\ (https://creativecommons.org/licenses/by-nc/4.0/). See http://ivyspring.com/terms for full terms and conditions.
}

Received: 2018.04.13; Accepted: 2019.01.04; Published: 2019.01.29

\begin{abstract}
Purpose: Trophinin-associated protein (TROAP) is a cytoplasmic protein that plays a significant role in the processes of embryo transplantation and microtubule regulation. However, the relevant survival analysis and cancer progression analysis have not yet been reported.

Methods: Eighteen matched pairs of tumor and adjacent non-tumor samples were evaluated to detect the TROAP mRNA level. Immunohistochemistry (IHC) was used to evaluate the TROAP expression in 108 hepatocellular carcinoma patients who underwent surgical resection. Meanwhile, data from the TCGA database was statistically evaluated.

Results: In the present study, we detected a significant increase in the TROAP mRNA level in tumor tissues when compared with adjacent non-tumor tissues. Moreover, the upregulation of TROAP was associated with increased serum AFP and GGT; the greater the tumor number was, the larger the tumor size, differentiation grade, and cancer embolus in clinical analysis. In HCC patients, elevated TROAP expression in the primary tumor was positively related to clinical severity, such as poor overall survival and disease-free survival. In addition, both univariate and multivariate survival analysis validated that TROAP expression was a promising independent risk factor for overall survival and disease-free survival in HCC patients. Furthermore, the results derived from the analysis of data from the TCGA database were consistent with previous results. Altogether, our results show that TROAP is a novel crucial regulator of HCC progression and is a potential therapeutic biomarker for HCC patients.
\end{abstract}

Conclusions: Elevated TROAP expression predicted a poor prognosis, and TROAP may serve as a potential biomarker for application in oncotherapy.

Key words: trophinin-associated protein, hepatocellular carcinoma, prognosis, and biomarker.

\section{Introduction}

Hepatocellular carcinoma (HCC) is one of the most common primary malignancies of cancer, and the third leading cause of cancer-related mortality worldwide. In Asia, half of patients come from China, and hepatitis $B$ virus (HBV) infection is the main etiologic factor for HCC [1-3]. It is well known that the 
prognosis of HCC remains poor due to its rapid development and metastasis [4-6]. Although there are many methods apply to treat it, such as surgical resection and orthotopic liver transplantation [7], a large number of patients still miss the opportunity to carry out curative resection as they present with an early tumor stage. Therefore, the key to treating HCC and achieving good outcomes is early detection and diagnosis.

Currently, serum alpha-fetoprotein (AFP), imaging technologies, and histology are three predominant methods for HCC screening [8]. However, only using AFP assay without typical AFP concentration has low sensitivity and specificity in patients. Imaging technologies can only diagnose tumors effectively when the diameter is greater than 1 $\mathrm{cm}$. Diagnostic histology depends on tissue biopsy, and it can largely improve the risk of the implantation metastasis [9-11]. Thus, it is urgent to find a valuable biomarker to monitor tumor progress and recurrence.

Trophinin-associated protein (TROAP, named TASTIN as well), a cytoplasmic protein composed of 778 amino acid residues, is proline-rich and contains src homology three domains [12]. Human TROAP is encoded by the TROAP gene, which maps to chromosome 12q13.12 and encodes an $83 \mathrm{kDa}$ protein [12-15]. TROAP is involved with bystin and trophinin in a cell adhesion molecule, and the trophinin-cell-adhesion molecule complex mediates an initial attachment of the blastocyst to uterine epithelial cells [13, 14, 16]. Notably, it is strongly expressed in cells and is also involved in embryo implantation and early placental development in humans [17-19]. Some reports demonstrated that TROAP expression enhances malignancy and promotes tumor development in breast cancer, colorectal cancer, ovarian adenocarcinomas, bladder urothelial carcinoma and other cancers. Moreover, TROAP has played a crucial role in HCC progression [20-22]. Nevertheless, how TROAP is involved in HCC recurrence and metastasis remains unclear.

Currently, the exploration of more effective targets is needed to better improve HCC patients' chances of survival. In this study, we aimed to document the clinical significance of TROAP expression and reveal the potential biomarkers of poor prognosis in HCC patients to identify a novel therapeutic target for HCC patients.

\section{Materials and methods}

\section{Patients and specimens}

Our study cohort was composed of 108 formalin-fixed, paraffin-embedded human HCC tissues and corresponding non-cancerous tissues from patients with hepatocellular carcinoma who had undergone surgical resection between 2010 and 2016; the mean age was 49.5 , and the study included 98 men and 10 women. The patients were followed up until December 2016. No patients had received other treatments such as chemotherapy, radiotherapy, or immunotherapy before the surgical resection. The available clinical data, such as gender, age, tumor number, tumor size, hepatitis B virus antigen, serum AFP, vascular invasion, liver cirrhosis, differentiation grade, and cancer embolus, were recorded for further analysis. The clinicopathological patient characteristics are summarized in Table 1.

Eighteen matched human HCC tissues and adjacent non-cancerous tissues were collected by quantitative real-time PCR analysis, and these samples were obtained from patients with HCC during May and July in 2016. Tissues were stored immediately in liquid nitrogen after surgical resection for the following analysis.

Time to recurrence (TTR) or disease-free survival (DFS) was calculated as the time between surgery and the detected recurrence, including detecting the abnormalities of tumor markers. Overall survival (OS) was defined as the interval from death or the last observation point.

All of our studies obtained approval from the research ethics committee at Sun Yat-Sen University Cancer Center.

\section{Real-time quantitative PCR analysis}

The expression level of the gene was determined by RT-PCR. The total mRNA of liver tumor tissues was isolated using Trizol reagent (Invitrogen, CA, USA). The internal control in our study used to measure the TROAP expression level was GAPDH. The relative expression levels of the target genes were estimated by two power values of $\Delta \mathrm{Ct}$ (the $\mathrm{Ct}$ of GAPDH minus the $\mathrm{Ct}$ of the target gene), and the experiments were repeated three times [23]. The PCR primer sequences were used in our research as follows:

TROAP, forward, 5' -TAAGGTCACTGGAGGGT TCTGG-3',

TROAP, reverse, 5'-CCTCTACTTCCTGTCTCA ACAGC-3',

GAPDH, forward, 5'-GTCTCCTCTGACTTCAA CAGCG-3',

GAPDH, reverse, 5' - ACCACCCTGTTGCTGTA GCCAA-3'.

\section{Immunohistochemistry}

TROAP expression in HCC tissues and matched adjacent non-cancerous tissues was evaluated by immunohistochemistry. We cut paraffin-embedded 
tissue samples into 4-5-um sections, which were deparaffinized and boiled in $0.1 \mathrm{M}$ citrate buffer (PH6.0) for 20 minutes to retrieve the antigen. Endogenous peroxidases were inactivated with 3\% hydrogen peroxide for 10 minutes. Then, the primary antibody TROAP (HPA044102, dilution 1:100, Sigma, USA) was incubated at $4^{\circ} \mathrm{C}$ overnight. Sections were washed with phosphate buffered saline and incubated with biotinylated secondary antibody for 30 minutes at room temperature. Next, 3, 3'-diaminobenzidine was applied for 5 minutes. Slides were counterstained with light hematoxylin, dehydrated, and cover-slipped.

We graded the level of TROAP by evaluating the staining intensity and percentage of cells stained. According to the staining intensity, we classified TROAP protein staining into four levels: absent expression, weak expression, moderate expression and strong expression; the score was labeled $0,1,2$, and 3 , respectively. In addition, we determined the percentage of positive tumor cell staining with a score of $0-100$. The weighted score for each case was calculated by multiplying the score of staining intensity and the percentage of positive tumor cell staining. In principle, the weighted scores ranged from $0(0 \%$ of cells staining $)$ to $3(100 \times 3 / 100)$. Furthermore, we characterized a score of $\leq 0.80$ as "low expression of TROAP" $(\mathrm{n}=51)$ and a score of $>0.80$ as "high expression of TROAP" $(n=57)$. IHC assessments were carried by two experienced pathologists in a double-blind manner. The IHC pictures were captured with an Olympus BX41 microscope (Olympus Optical, Japan) at 100 magnifications and 200 magnifications.

\section{The cancer genome atlas (TCGA) data analysis}

Publicly available human HCC datasets from TCGA were analyzed. The mRNA expression profiles downloaded from the TCGA portal (https://cancergenome.nih.gov/) included 48 normal and 367 tissue samples.

\section{Statistical analysis}

All data in this study were evaluated with SPSS 21.0 software (SPSS Inc., Chicago, USA) and carried out using GraphPad Prism (GraphPad software). All data are shown as the mean \pm standard deviation. The results of real-time quantitative PCR were determined using Student's t-test. Survival analysis was demonstrated using the Kaplan-Meier method and log-rank tests. The relevance between TROAP expression and clinicopathological parameters was assessed by the chi-square test. A Cox proportional hazards regression model was constructed to evaluate, and $p<0.05$ was considered to indicate statistically significant differences.

\section{Results}

\section{TROAP is up-regulated in HCC tissues}

The possible role of TROAP in hepatocellular carcinomas was determined using real-time quantitative PCR assay to investigate the gene expression in 18 pairs of tumor tissues and adjacent non-cancerous tissues. Compared to the expression of TROAP in adjacent non-cancerous tissues, TROAP was highly expressed in liver cancer tissues (Figure 1A), 13 of 18 tissues were detected that TROAP was up-regulated (Figure 1C). Based on real-time quantitative PCR, the receiver operating characteristic (ROC) curves were used to estimate the value of TROAP mRNA expression, yielding an area under the curve (AUC) of 0.711 (Figure 1D). Similar results from the TCGA analysis supported that conclusion. The expression of TROAP was significantly upregulated in 48 pairs of tumor tissues (Figure 1B), yielding area under the curve (AUC) of 0.965 (Figure 1E), n=415.

\section{High expression of TROAP is associated with aggressive clinicopathological parameters in HCC patients}

As increased TROAP mRNA expression in tumor tissues has been proven, it is necessary to elucidate the clinical relevance of TROAP expression. To further determine whether the higher expression of TROAP was associated with poor progression in HCC patients after undergoing surgical resection, 108 specimens were analyzed by immunohistochemistry essay. As shown in Figure 2, TROAP expression was mainly located in the cytoplasm, and 47\% (51 of 108) of HCC patients stained for low TROAP expression, and the remaining $53 \%$ (57 of 108) of HCC patients stained for high TROAP expression. The clinical characteristics of these patients are shown in Table 1. Interestingly, five clinicopathological parameters are relevant to high TROAP expression, including serum AFP level, tumor size, tumor number, differentiation grade, and cancer embolus.

We also analyzed the AFP, GGT, CA50, CA19-9 and CEA levels of the two groups (the TROAP positive group and the TROAP negative group), and serum AFP (13090 \pm 4021) and GGT (97.27 \pm 13.69) were higher in the TROAP-positive group. In contrast, AFP $(3146 \pm 1750)$ and GGT $(58.33 \pm 5.568)$ were expressed less in the TROAP-negative group (Figure 3A). Meanwhile, there was no difference in the levels of CEA $(2.249 \pm 0.215$ vs $2.350 \pm 0.212)$, CA50 $(24.85 \pm 10.42$ vs. $10.60 \pm 4.562)$ and CA19-9 (26.86 \pm 4.426 vs. $30.12 \pm 6.827$ ) (Figures 3C, D, E). 
Interestingly, the expression of TROAP and the serum AFP showed a positive correlation $(p=0.0018$, $\mathrm{R}=0.2971$; Figure 3F).

\section{Up-regulation of the TROAP protein is associated with poor prognosis in HCC patients}

To further explore the relationship between TROAP expression and survival, we evaluated overall survival and disease-free survival with Kaplan-Meier survival analysis. Obviously, the results displayed

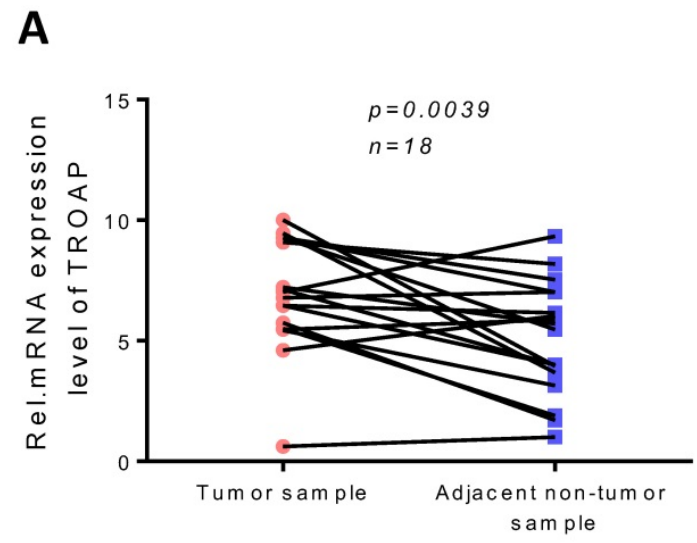

B
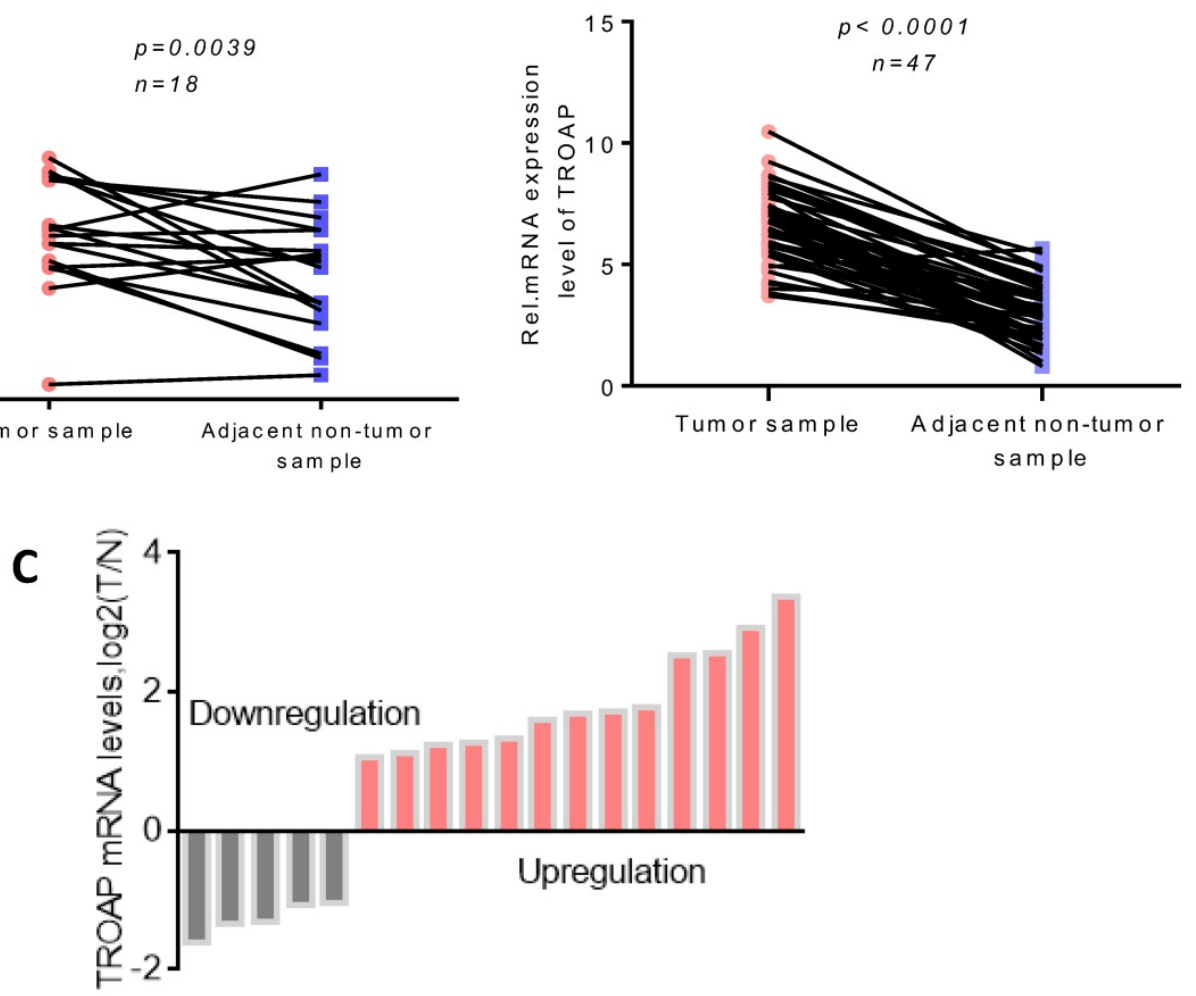

D

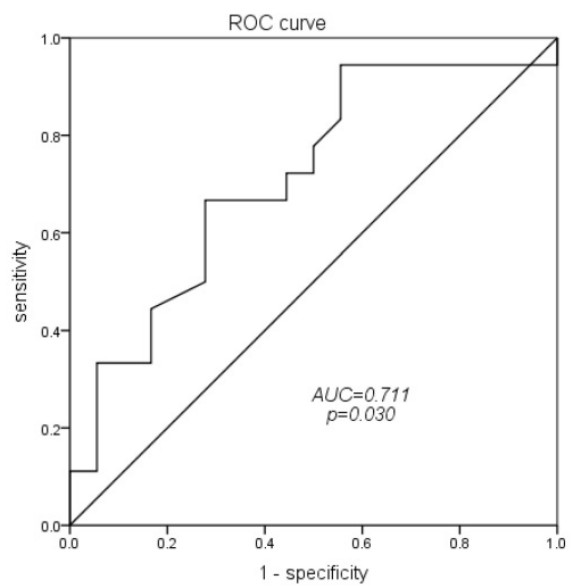

E



Figure 1. TROAP is up-regulation in HCC tissues. In our data (A) and TCGA data (B), TROAP mRNA expression in HCC tissues are significantly higher than adjacent non-cancerous tissues. (C)TROAP mRNA expression in HCC tumor tissues and adjacent non-cancerous tissues, TROAP is up-regulation in 13 of 18 . In our data (C) and TCGA data (D), non-cancerous tissues ROC curve was constructed according to TROAP expression in HCC tissues and non-cancerous tissues. 


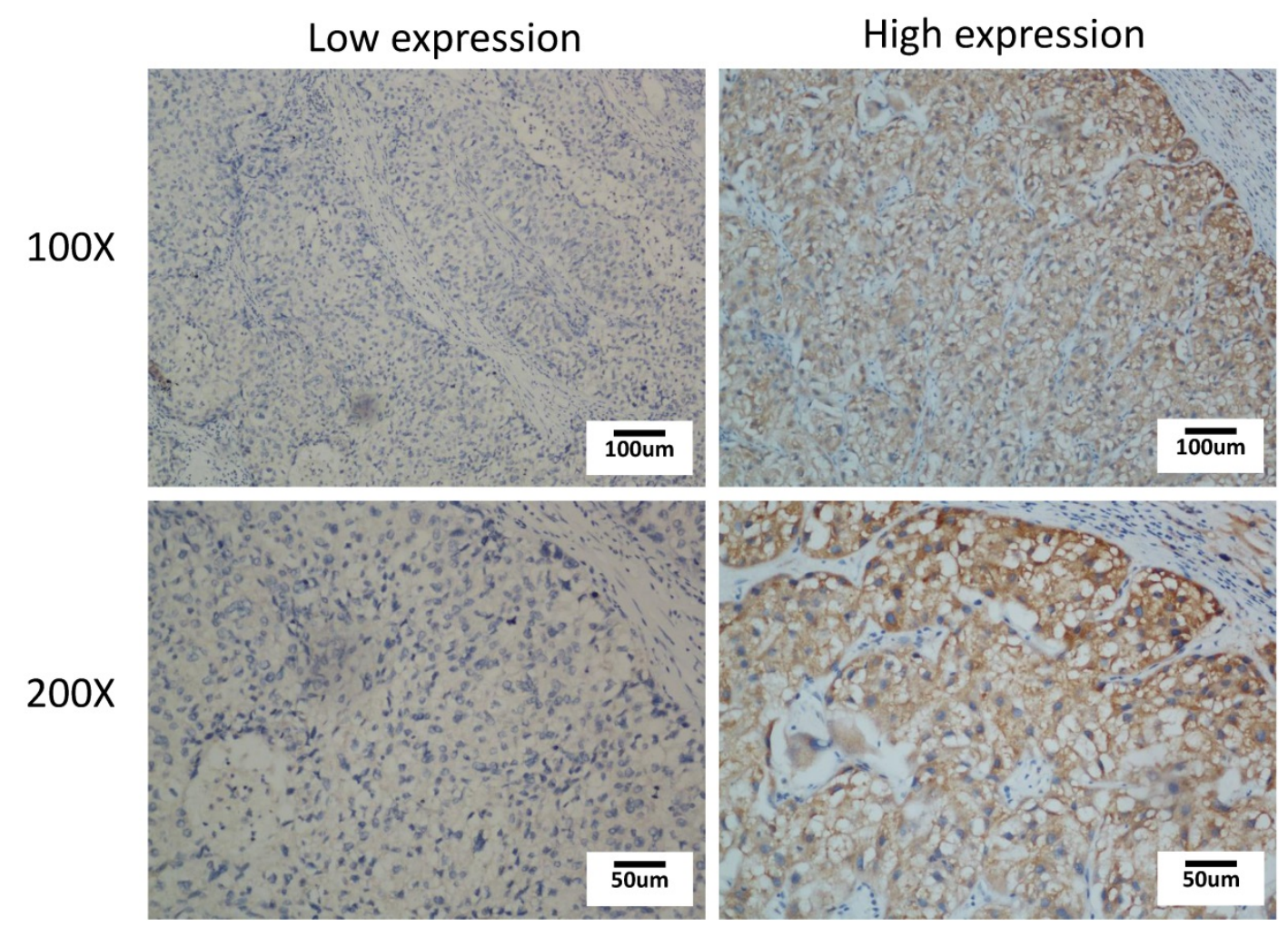

Figure 2. Immunohistochemical staining of TROAP expression in HCC tissue with low expression and high expression

Table 1. Correlation of TROAP expression with clinicopathological features in HCC

\begin{tabular}{|c|c|c|c|c|}
\hline \multirow[t]{2}{*}{ Characteristics } & \multirow[t]{2}{*}{ Cases } & \multicolumn{2}{|c|}{ TROAP expression } & \multirow[t]{2}{*}{$p$ value } \\
\hline & & $\begin{array}{l}\text { High expression } \\
(\mathrm{n}=57)\end{array}$ & $\begin{array}{l}\text { Low expression } \\
(\mathrm{n}=51)\end{array}$ & \\
\hline Gender & & & & 0.328 \\
\hline Female & 10 & 7 & 3 & \\
\hline Male & 98 & 50 & 48 & \\
\hline Age, years & & & & 0.568 \\
\hline$<=50$ & 56 & 28 & 28 & \\
\hline$>50$ & 52 & 29 & 23 & \\
\hline HBsAg & & & & 0.999 \\
\hline Negative & 10 & 5 & 5 & \\
\hline Positive & 98 & 52 & 46 & \\
\hline Serum AFP, ug/l & & & & $0.011^{*}$ \\
\hline$<=200$ & 64 & 27 & 37 & \\
\hline$>200$ & 44 & 30 & 14 & \\
\hline Tumor Number & & & & $0.011^{*}$ \\
\hline Single & 83 & 38 & 45 & \\
\hline Multiple & 25 & 19 & 6 & \\
\hline Tumor Size, $\mathrm{cm}$ & & & & $0.004^{*}$ \\
\hline$<=5$ & 71 & 30 & 41 & \\
\hline$>5$ & 37 & 27 & 10 & \\
\hline Vascular invasion & & & & 0.055 \\
\hline No & 21 & 7 & 14 & \\
\hline Yes & 87 & 50 & 37 & \\
\hline Liver cirrhosis & & & & 0.842 \\
\hline Absent & 37 & 19 & 18 & \\
\hline Present & 71 & 38 & 33 & \\
\hline Differentiation grade & & & & $0.000^{*}$ \\
\hline $\mathrm{I} / \mathrm{II}$ & 50 & 22 & 38 & \\
\hline III/IV & 48 & 35 & 13 & \\
\hline Cancer embolus & & & & \\
\hline No & 97 & 46 & 50 & $0.005^{*}$ \\
\hline Yes & 12 & 11 & 1 & \\
\hline
\end{tabular}

We found that only 12 of $51(23.53 \%)$ patients died in the low TROAP expression group (mean: $2083 \pm 41.51$ days), while 34 of $57(59.65 \%)$ patients with high TROAP expression died (mean: $1403 \pm 99.01$ days), (Figure 5A, C). Similarly, among 51 patients, only $30(58.82 \%)$ had recurrence with low TROAP expression, while 46 of $57(80.70 \%)$ patients had recurrence with high TROAP expression (Figure 5B). The mean time of recurrence after undergoing surgery in the low and high TROAP expression groups were $1386 \pm 126.0$ days and $593.6 \pm 102.8$ days, respectively (Figure 5D). Data derived from the TCGA database showed similar results (Figure 5E, F). Moreover, the TROAP expression is relevant to the $\mathrm{T}$ stage and overall stage, but there was no significant relevance between TROAP expression and N or M stage (Figure 6).

This demonstrated that the high expression of TROAP is associated with a high risk of recurrence and death, while low expression of TROAP is associated with a significantly longer overall survival time and disease-free survival time. The high expression of TROAP can result in poor survival in HCC patients.

\section{TROAP plays an independent prognostic role in liver cancer}

To investigate whether TROAP is an independent factor in liver cancer in the entire study 
population, univariate and multivariate Cox analysis was conducted using SPSS version 21, Table 2 presents the results. The univariate analysis showed that there are five factors such as age, tumor size, tumor number and lymph node metastasis. TROAP expression was a poor prognostic factor for OS in liver cancer patients. According to multivariate analysis, these three factors such as age, tumor number, and TROAP expression were independent risk markers associated with shorter overall survival. The similar method was used to analyze disease-free survival, age, tumor size, tumor number, lymph node metastasis and TROAP staining, those five factors as independent predictors for prognosis by univariate analysis. Multivariate Cox regression analysis indicated that age, tumor number, and TROAP expression might play an independent role for poor disease-free survival.

\section{Discussion}

Hepatocellular carcinoma is one the most malignant tumors worldwide; almost 50 percent of those with this carcinoma are living in China, and HBV infection is the first risk factor [3, 24, 25]. From our data, most of the patients were infected with HBV (98 of 108) as well. Due to the poor prognosis and recurrence, $\mathrm{HCC}$ has the 3rd highest mortality rate of all cancers [26, 27]. Numerous biomarkers have been reported in recent years, but there is no effective biomarker for clinical diagnosis [28, 29]. It is urgently necessary to identify a reliable biomarker to monitor the progression of the tumor.
A

\begin{tabular}{|c|c|c|}
\hline \multirow{2}{*}{} & \multicolumn{2}{|c|}{ TROAP expression } \\
\cline { 2 - 3 } & Low & High \\
\hline CA19-9(U/ml) & $30.12 \pm 6.827$ & $26.86 \pm 4.426$ \\
\hline CEA(ng/ml) & $2.35 \pm 0.212$ & $2.249 \pm 0.215$ \\
\hline AFP(ng/ml) & $3146 \pm 1750$ & $13090 \pm 4021^{*}$ \\
\hline CA50(IU/mL) & $10.60 \pm 4.562$ & $24.85 \pm 10.42$ \\
\hline GGT(u/L) & $58.33 \pm 5.568$ & $97.27 \pm 13.63^{*}$ \\
\hline
\end{tabular}

B

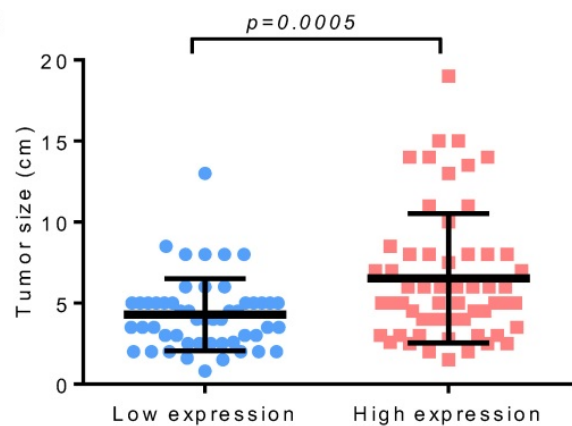

C

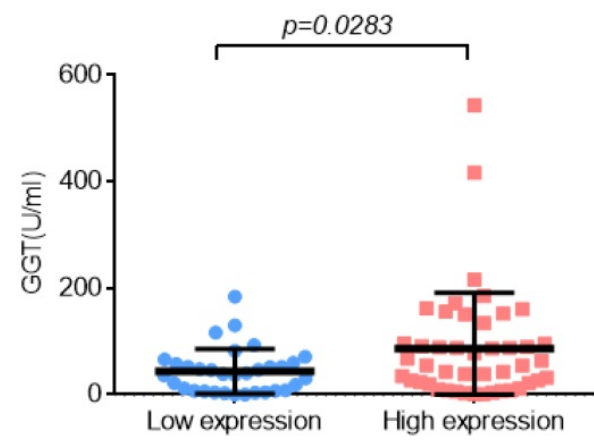

E

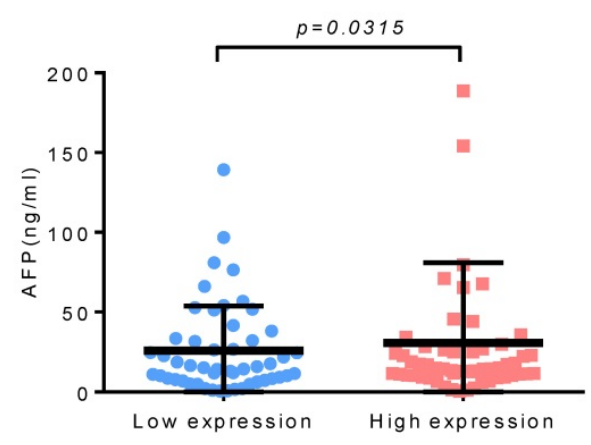

D

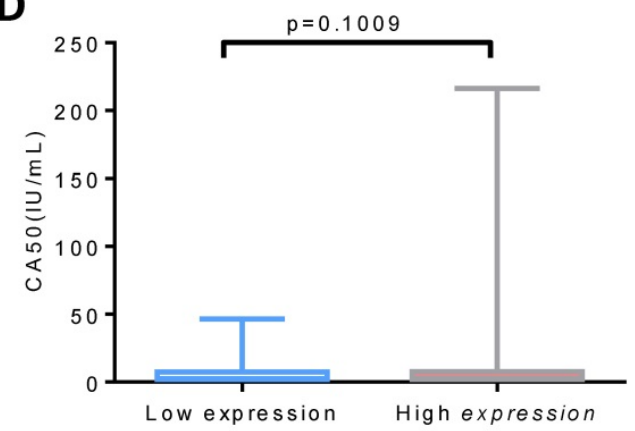

$\mathbf{F}$

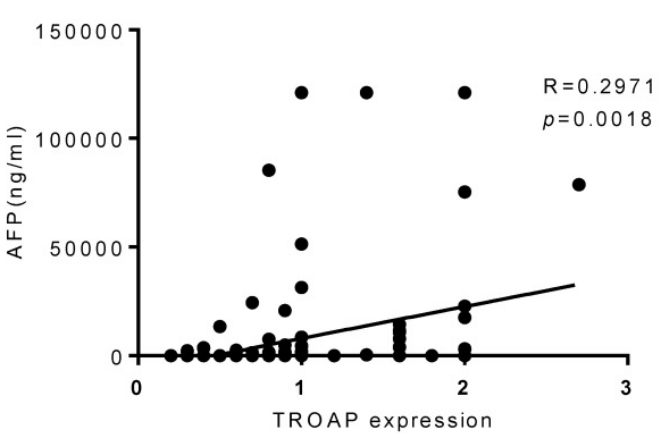

Figure 3. The TROAP expression is associated with some clinical parameters, (A) Serum tumor marker. (B) Tumor size. (C) The serum level of GGT, (E) AFP, (A, D) but no association between CA19-9, CEA, CA50. (F) The positive correlation between the level of serum AFP and level of TROAP expression in HCC patients' tissues. 
A

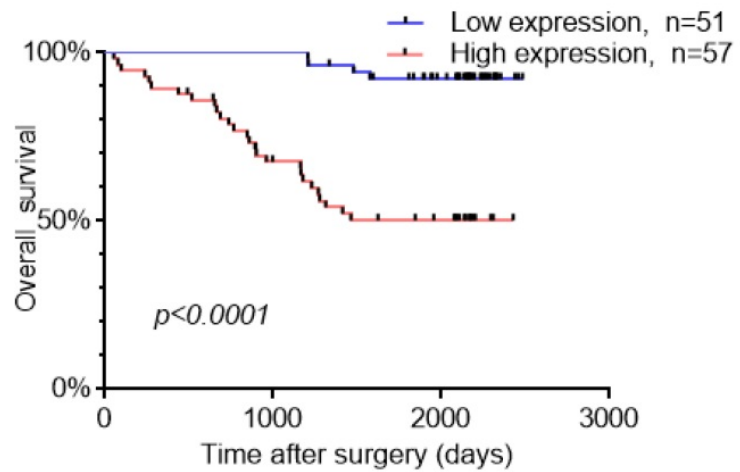

B

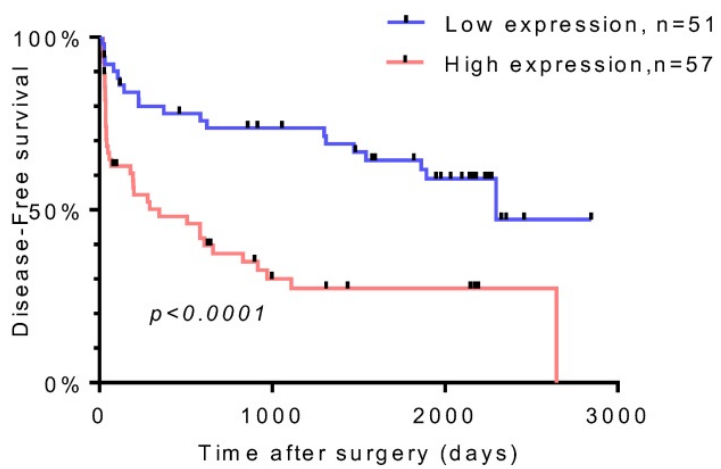

C

\begin{tabular}{|c|c|c|c|c|}
\hline \multirow{2}{*}{} & \multicolumn{2}{|c|}{ Low expression } & \multicolumn{2}{c|}{ High expression } \\
\cline { 2 - 5 } & OS & DFS & OS & DFS \\
\hline 3 years & $98.04 \%$ & $61.76 \%$ & $73.69 \%$ & $30.05 \%$ \\
\hline 5 years & $92.07 \%$ & $50.18 \%$ & $64.40 \%$ & $27.32 \%$ \\
\hline
\end{tabular}

D

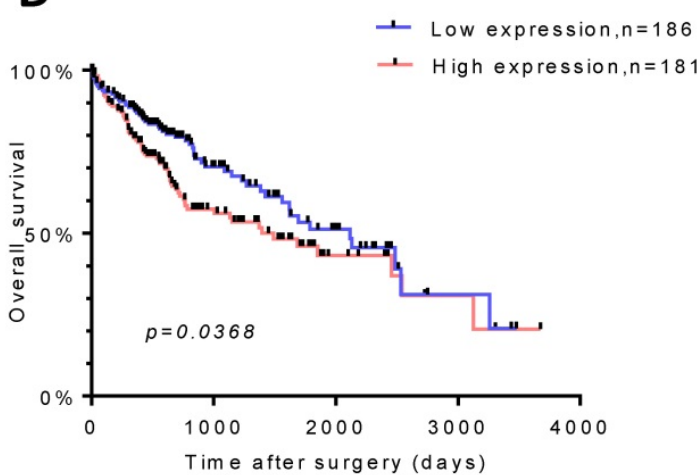

E

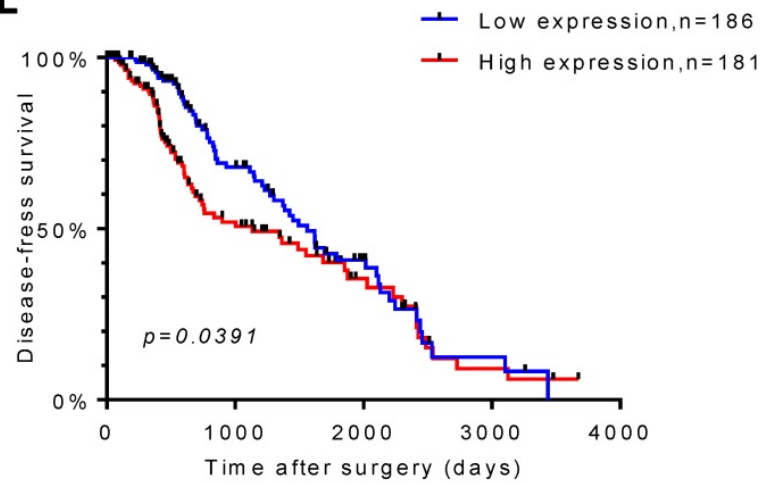

Figure 4. TROAP expression is related to HCC patient survival. Kaplan-Meier analysis for overall survival (A) and diseased-free survival (B) for patients with HCC stratified. (C) Rate of the OS and DSF in 3and 5 years respectively. In TCGA data, Kaplan-Meier analysis for overall survival (D) and diseased-free survival (E)

TROAP's major function is to maintain the structural and dynamic features of centrosomes during mitosis $[15,16]$. In cancer cells, due to the TROAP overexpression to genomic imbalance, normal spindle structure would be disrupted [12]. Studies have shown that TROAP is necessary for trophinin to function as a cell adhesion molecule, which could influence tumor progression $[18,30]$. Several studies have proven that TROAP was overexpressed in a series of cancer types and was implicated in cell proliferation and metastasis [21, 22, 31]. Hu et al. [32] has been reported that TROAP may play a negative role in prognosis in breast cancer, head and neck squamous, non-small cell lung carcinoma and other 13 cancer types by bioinformatics method to explore the data from TCGA. They suggested that the growth of cancer cells was inhibited with TROAP downregulation.

Interestingly, Lian et al. [33] implied that TROAP may suppress HCC cellular growth and migration in vitro. They also demonstrated that their data was contradictory to the TCGA data because their HCC sample origins were from $\mathrm{HBV}$-infected patients, so the research mainly explored the relationship between the TROAP and HBV-infected patients. An early event of the HBV-infected patients, HBV DNA can 
integrate into the host genome, and the host genome will induce instability and eventually insertional mutagenesis directly [34]. Although the primary mechanism of development of HBV-infected HCC patients is unclear yet, various studies have demonstrated that a high rate of chromosomal alterations, p53 inactivation, and overexpression of fetal liver/hepatic progenitor cells genes might be responsible for HBV-related HCCs. However, many factors can cause the HCC initiation and development, including HBV, HCV, alcohol, aflatoxin B1, and other environmental factors. In our data shown above and the data from the Cancer Genome Atlas, HBV-infected patients are not the majority. The opposite results may suggest that the mechanisms of HCC initiation and development between non-HBV-infected and HBV-infected patients have a considerable difference. We suggest the role of TROAP in patients' prognosis after surgical treatment
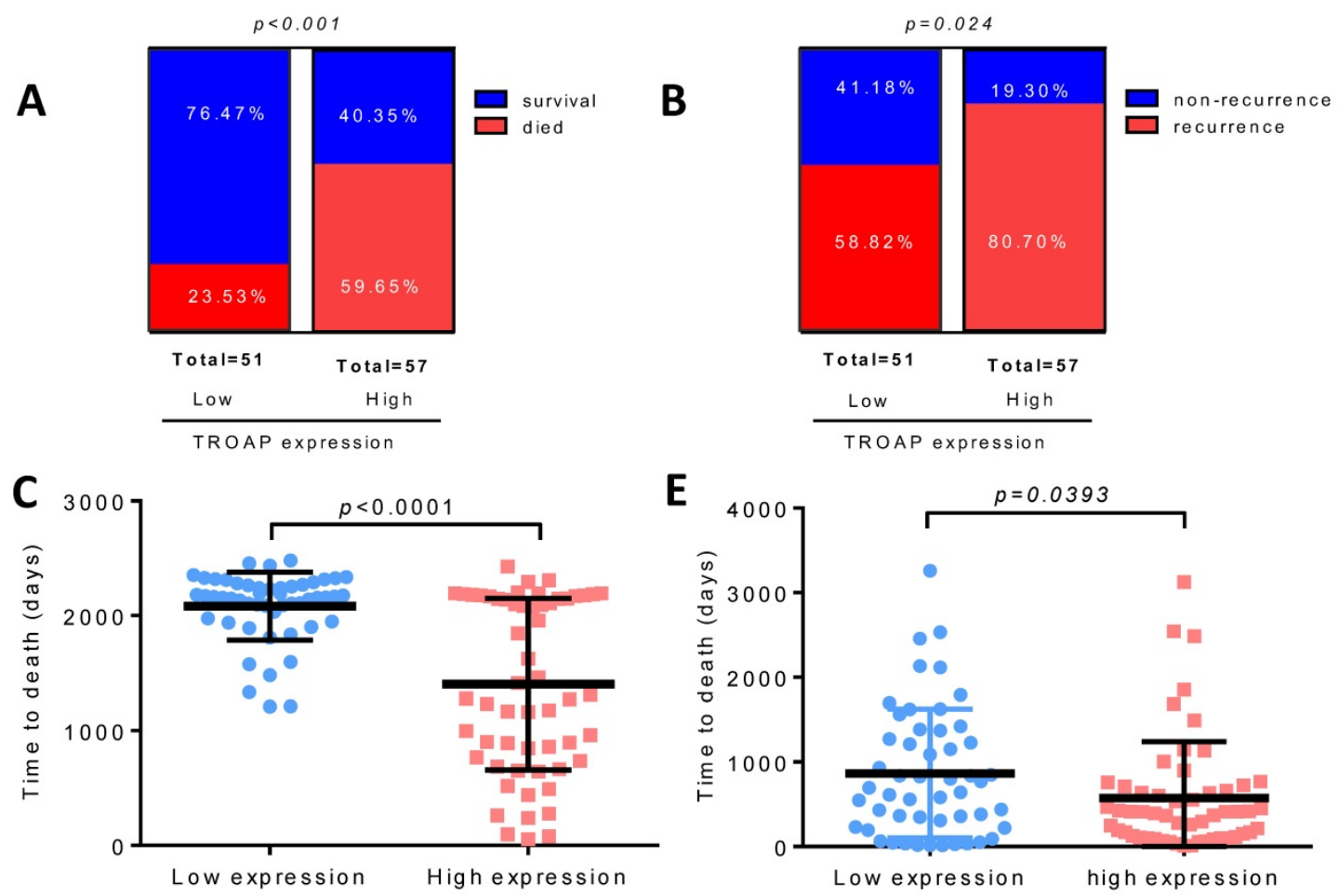

D

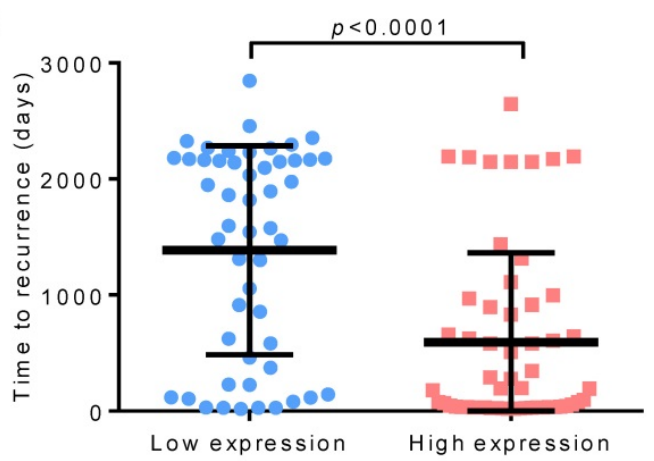

for the first time. We found that the expression of TROAP was highly expressed in HCC tissues than in corresponding adjacent non-tumor tissues. In addition, the high expression of TROAP is highly correlated with a larger tumor size and a higher number of tumors than the low expression of TROAP. Similar results were observed in database analysis. At the same time, comparing the low expression of TROAP, the levels of AFP and GGT in serum were significantly higher, and there was no association with CA19-9, CEA, and CA50. Many papers have reported that the 5-year survival rate ranged from $50 \%$ to $70 \%$; however, in this cohort study, the overall survival rate was $78.70 \%$ in the entire population. There was a lower rate of overall survival with high TROAP expression at 5 years $(50.18 \%)$, while the rate of disease-free survival was $42.86 \%$ in the entire population and $27.32 \%$ in the high TROAP expression group.

\section{$\mathbf{F}$}

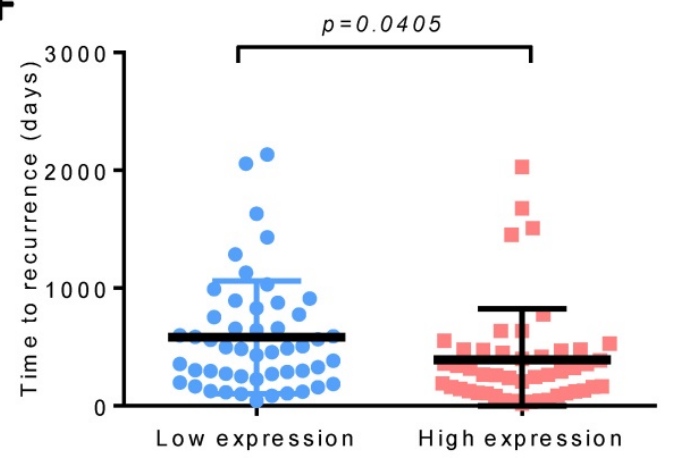

Figure 5. High TROAP expression is associated with shortened survival of HCC patients. Higher level of TROAP is associated with higher rate of died (A) and recurrence (B), shorter time to death (C) and to recurrence (D). Similar results in TCGA (E, F). 

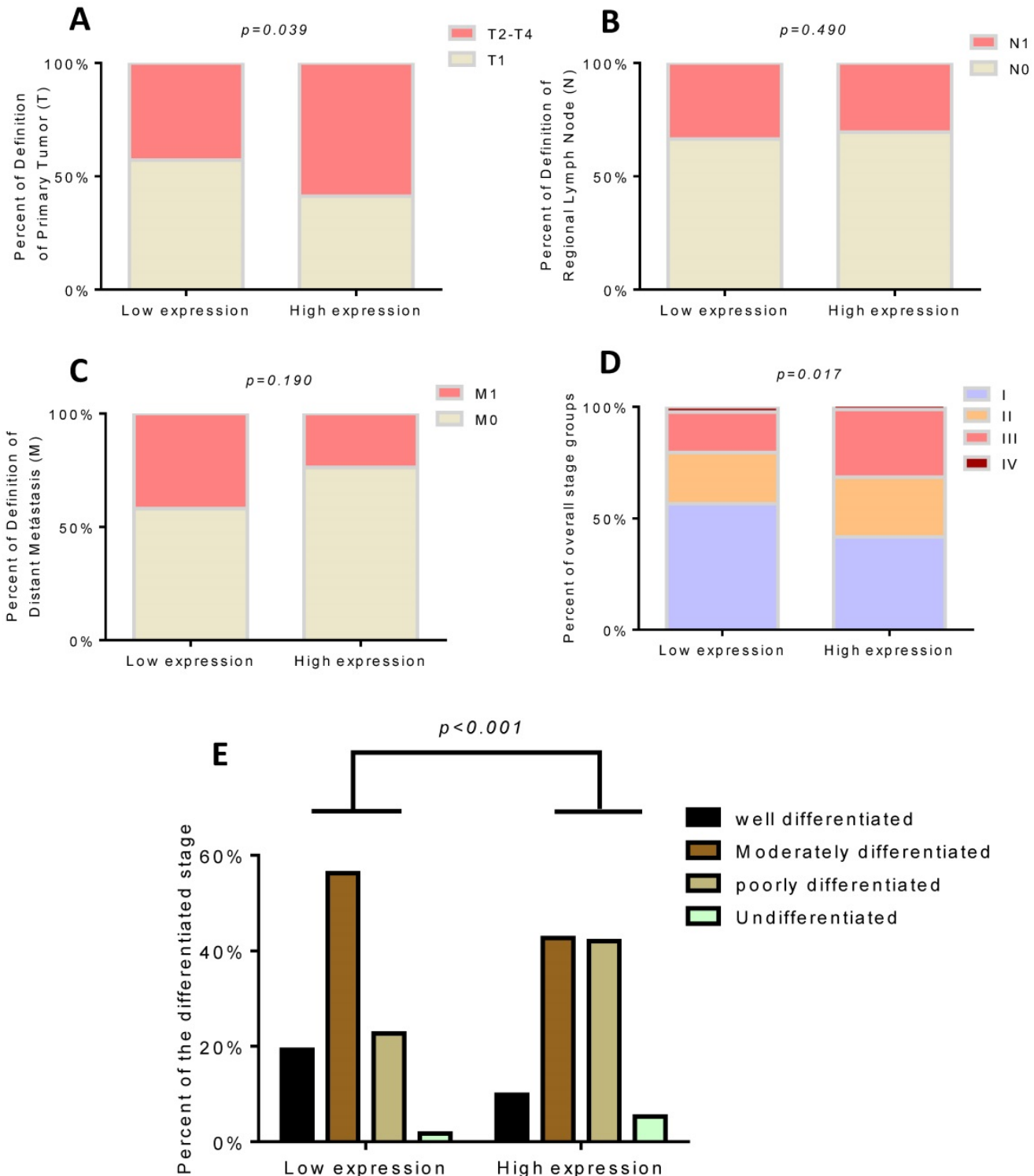

Figure 6. High TROAP expression is associated with $T$ stage $(A)$ and overall stage (D), but no association with $N$ or $M$ stage (B, C). The expression of TROAP also is related to tissue differentiation $(\mathrm{E})$.

Table 2. Univariate and multivariate COX analysis for TROAP and survival of HCCs

\begin{tabular}{lllll}
\hline Parameters & OS & \multicolumn{2}{l}{ DFS } & \\
\cline { 2 - 5 } & HR $(95 \% \mathrm{CI})$ & $p$ & HR $(95 \% \mathrm{CI})$ & $p$ \\
\hline Univariate analysis & & & & \\
Gender, male vs female & $0.42(0.16-1.09)$ & 0.073 & $0.45(0.17-1.17)$ & 0.099 \\
Age, years, $<=50 \mathrm{vs}>50$ & $2.15(1.02-4.49)$ & $\mathbf{0 . 0 4 2}$ & $2.11(1.04-4.30)$ & $\mathbf{0 . 0 0 4}$ \\
HBsAg, negative vs positive & $1.62(0.39-6.80)$ & 0.508 & $1.75(0.42-7.30)$ & 0.445 \\
AFP,ng/ml,>=200vs<200 & $1.63(0.81-3.30)$ & 0.174 & $1.44(0.72-2.85)$ & 0.300 \\
Cirrhosis, yes vs no & $0.93(0.45-1.94)$ & 0.847 & $0.76(0.39-1.56)$ & 0.474 \\
Tumor size, $>=5$ vs $<5 \mathrm{~cm}$ & $2.78(1.37-5.62)$ & $\mathbf{0 . 0 0 5}$ & $2.43(1.23-4.82)$ & $\mathbf{0 . 0 1 1}$ \\
Tumor number(multiple vs single) & $4.26(2.10-8.66)$ & $\mathbf{0 . 0 0 0}$ & $3.80(1.91-7.56)$ & $\mathbf{0 . 0 0 0}$ \\
Differentiation status, I/ii vs III/IV & $1.66(0.81-3.38)$ & 0.167 & $1.64(0.82-3.28)$ & 0.160 \\
Tumor thrombosis, yes vs no & $2.24(0.86-5.86)$ & 0.100 & $2.07(0.80-5.39)$ & 0.135 \\
Microvascular invasion, yes vs no & $1.05(0.43-2.55)$ & 0.922 & $1.14(0.47-2.76)$ & 0.774 \\
Lymph node metastasis, yes /no & $2.67(1.19-5.97)$ & $\mathbf{0 . 0 1 7}$ & $2.46(1.11-5,45)$ & $\mathbf{0 . 0 2 7}$ \\
TROAP, low vs high expression & $8.86(3.10-25.44)$ & $\mathbf{0 . 0 0 0}$ & $5, .89(2.43-14.32)$ & $\mathbf{0 . 0 0 0}$ \\
& & & & \\
Multivariate analysis & & & & \\
Age, years, $<=50 \mathrm{vs}>50$ & $2.43(1.02-5.74)$ & $\mathbf{0 . 0 4 4}$ & $2.40(1.05-5.48)$ & $\mathbf{0 . 0 3 8}$ \\
Tumor size, $>=5$ vs <5cm & $1.75(0.72-4.23)$ & 0.214 & $1.57(0.65-3.77)$ & 0.312 \\
Tumor number(multiple vs single) & $2.71(1.18-6.21)$ & $\mathbf{0 . 0 1 9}$ & $2.49(1.11-5.55)$ & $\mathbf{0 . 0 2 7}$ \\
\hline
\end{tabular}

\begin{tabular}{lllll}
\hline Parameters & OS & \multicolumn{2}{l}{ DFS } & \\
\cline { 2 - 5 } & $\mathrm{HR}(95 \% \mathrm{CI})$ & $p$ & $\mathrm{HR}(95 \% \mathrm{CI})$ & $p$ \\
\hline Lymph node metastasis, yes /no & $2.33(0.83-6.57)$ & 0.111 & $2.35(0.78-7.09)$ & 0.130 \\
TROAP, low vs high expression & $5.91(1.89-18.53)$ & $\mathbf{0 . 0 0 2}$ & $4.38(1.55-12.35)$ & $\mathbf{0 . 0 0 5}$ \\
\hline NOTE: The entire clinicopathological variables lists in the table were included in \\
the univariate and multivariate analysis. \\
Abbreviation: 95\%CI, 95\%confidence interval.
\end{tabular}

The greatest challenge in the treatment of HCC is early diagnosis. Although the sensitivity and specificity of AFP remain unsatisfactory, AFP is still the most frequent biomarker to diagnose and evaluate the prognosis of HCC $[35,36]$. In our research, the positive correlation between the level of AFP and TROAP protein was tested with the Pearson correlation test. Furthermore, 18 matched pairs of tissues from patients who have undergone surgical resection showed that TROAP was highly expressed in tumor tissues relative to adjacent non-tumorous 
tissues, to construct a receiver operating characteristic (ROC) curve, the ROC curve in our study suggested that TROAP expression had valid diagnostic value for HCC with the AUC of 0.711 , and the statistical power of that result is limited because the sample size was not large enough, and the same value of the AUC of 0.978 with the TCGA data analysis $(\mathrm{n}=415)$. Abdel et al.[37]predicted that the co-expression of TROAP and TOPO2A as a biomarker, which is related to anthracycline-based chemotherapy (ATC-CT) in breast cancer. TROAP expression may be a potential biomarker for this type of cancer. However, another limitation existed in our study, and whether the sensitivity and the specificity may enhance the statistical power by analyzing co-expression of the level of the serum AFP and the TROAP level of the liver, further exploration may carry out in the following research.

Using univariate analysis to explore clinicopathological characteristics, age, tumor size, tumor number, lymph node metastasis and TROAP expression, which are five high-risk factors associated with poor prognosis for both overall survival and disease-free survival. In addition, through multivariate analysis, age, tumor number, and TROAP expression these three factors may play a role in independent prognosis.

At the same time, the expression of TROAP is also associated with the differentiation grade of tumors. The function of the gene in the cell cycle [31] and its action as a cell adhesion molecule [38] has been reported, but none of them validated the relationship between differentiation and the protein level. However, the relevant molecular mechanism was not explored in our study in detail. Cheng et al. [39] demonstrated that the basal level change by the methylation of TROAP in HeLa cells and ES cells could promote cell proliferation and anti-apoptosis through NF-kB, TGF- $\beta$, and PI3K pathways using large databases and genome-wide high-throughput analysis.

Collectively, our research showed that TROAP was overexpressed in liver cancer patients may indicate a worse prognosis at first. Our study demonstrated an elevated TROAP expression level predicted a poorer prognosis, including an increased serum AFP and GGT level, the larger tumor size, the greater the tumor number, differentiation grade, cancer embolus, and the rate of death and recurrence. In addition, TROAP might be a novel biomarker for diagnosis and potential targets of therapy. Although our research has identified the relationship between the TROAP and patients' prognosis, there are also limitations. First, larger-scale samples are needed to elevate the statistical power and confirm these results.
Second, the detailed molecular mechanism is still unclear, further studies need to be performed.

In summary, we found that TROAP played a significant role in HCC prognosis, and TROAP is a candidate therapeutic biomarker for HCC patients.

\section{Abbreviations}

TROAP: trophinin-associated protein; IHC: immunohistochemistry; HCC: hepatocellular carcinoma; HBV: hepatitis B virus; AFP: alpha-fetoprotein; DFS: disease-free survival; OS: overall survival; TCGA: the Cancer Genome Atlas; ROC: receiver operating characteristic; AUC: area under the curve.

\section{Acknowledgments}

We thank Prof. Chao-Nan Qian for his help in the study. The research was supported by the National Natural Science Foundation of China (Grant No.81673903, 81373500, 81773162).

\section{Competing Interests}

The authors have declared that no competing interest exists.

\section{References}

1. El-Serag HB, Rudolph KL. Hepatocellular carcinoma: epidemiology and molecular carcinogenesis. Gastroenterology. 2007; 132: 2557-76.

2. Chen W, Zheng R, Baade PD, Zhang S, Zeng H, Bray F, et al. Cancer statistics in China, 2015. CA: a cancer journal for clinicians. 2016; 66: 115-32.

3. Liu L, Liu Y, Liu J, Zhai X, Wen J, Xie K, et al. Genetic variants in pseudogene E2F3P1 confer risk for HBV-related hepatocellular carcinoma in a Chinese population. Journal of biomedical research. 2013; 27: 215-9.

4. Yamashita T, Honda M, Nakamoto Y, Baba M, Nio K, Hara Y, et al. Discrete nature of EpCAM+ and CD90+ cancer stem cells in human hepatocellular carcinoma. Hepatology (Baltimore, Md). 2013; 57: 1484-97.

5. Pang RW, Joh JW, Johnson PJ, Monden M, Pawlik TM, Poon RT. Biology of hepatocellular carcinoma. Annals of surgical oncology. 2008; 15: 962-71.

6. Bruix J, Llovet JM. Major achievements in hepatocellular carcinoma. Lancet (London, England). 2009; 373: 614-6.

7. Zhu ZX, Huang JW, Liao MH, Zeng Y. Treatment strategy for hepatocellular carcinoma in China: radiofrequency ablation versus liver resection. Japanese journal of clinical oncology. 2016; 46: 1075-80.

8. Benson AB, 3rd, Abrams TA, Ben-Josef E, Bloomston PM, Botha JF, Clary BM, et al. NCCN clinical practice guidelines in oncology: hepatobiliary cancers. Journal of the National Comprehensive Cancer Network : JNCCN. 2009; 7: 350-91.

9. Chisari FV, Ferrari C. Hepatitis B virus immunopathogenesis. Annual review of immunology. 1995; 13: 29-60.

10. Maluccio M, Covey A. Recent progress in understanding, diagnosing, and treating hepatocellular carcinoma. CA: a cancer journal for clinicians. 2012; 62: 394-9.

11. Farinati F, Marino D, De Giorgio M, Baldan A, Cantarini M, Cursaro C, et al. Diagnostic and prognostic role of alpha-fetoprotein in hepatocellular carcinoma: both or neither? The American journal of gastroenterology. 2006; 101: 524-32.

12. Yang S, Liu X, Yin Y, Fukuda MN, Zhou J. Tastin is required for bipolar spindle assembly and centrosome integrity during mitosis. FASEB journal : official publication of the Federation of American Societies for Experimental Biology. 2008; 22: 1960-72.

13. Nadano D, Nakayama J, Matsuzawa S, Sato TA, Matsuda T, Fukuda MN. Human tastin, a proline-rich cytoplasmic protein, associates with the microtubular cytoskeleton. The Biochemical journal. 2002; 364: 669-77.

14. Suzuki N, Zara J, Sato T, Ong E, Bakhiet N, Oshima RG, et al. A cytoplasmic protein, bystin, interacts with trophinin, tastin, and cytokeratin and may be involved in trophinin-mediated cell adhesion between trophoblast and endometrial epithelial cells. Proceedings of the National Academy of Sciences of the United States of America. 1998; 95: 5027-32.

15. Nakayama J, Aoki D, Suga T, Akama TO, Ishizone S, Yamaguchi $\mathrm{H}$, et al. Implantation-dependent expression of trophinin by maternal fallopian tube 
epithelia during tubal pregnancies: possible role of human chorionic gonadotrophin on ectopic pregnancy. The American journal of pathology. 2003; 163: 2211-9.

16. Fukuda MN, Sugihara K. Trophinin in cell adhesion and signal transduction. Frontiers in bioscience (Elite edition). 2012; 4: 342-50.

17. Fukuda MN. Molecular basis of embryo implantation. The Keio journal of medicine. 1996; 45: 37-43.

18. Fukuda MN, Sato T, Nakayama J, Klier G, Mikami M, Aoki D, et al. Trophinin and tastin, a novel cell adhesion molecule complex with potential involvement in embryo implantation. Genes \& development. 1995; 9: 1199-210.

19. Aoki R, Fukuda MN. Recent molecular approaches to elucidate the mechanism of embryo implantation: trophinin, bystin, and tastin as molecules involved in the initial attachment of blastocysts to the uterus in humans. Seminars in reproductive medicine. 2000; 18: 265-71.

20. Partheen K, Levan K, Osterberg L, Claesson I, Fallenius G, Sundfeldt K, et al. Four potential biomarkers as prognostic factors in stage III serous ovarian adenocarcinomas. International journal of cancer. 2008; 123: 2130-7.

21. Godoy H, Mhawech-Fauceglia P, Beck A, Miliotto A, Miller A, Lele S, et al. Developmentally restricted differentiation antigens are targets for immunotherapy in epithelial ovarian carcinoma. International journal of gynecological pathology : official journal of the International Society of Gynecological Pathologists. 2013; 32: 536-40.

22. Harada O, Suga T, Suzuki T, Nakamoto K, Kobayashi M, Nomiyama T, et al. The role of trophinin, an adhesion molecule unique to human trophoblasts, in progression of colorectal cancer. International journal of cancer. 2007; 121: $1072-8$.

23. Xu L, Huang TJ, Hu H, Wang MY, Shi SM, Yang Q, et al. The developmental transcription factor IRF6 attenuates ABCG2 gene expression and distinctively reverses stemness phenotype in nasopharyngeal carcinoma. Cancer letters. 2018; 431: 230-43.

24. Ringelhan $\mathrm{M}, \mathrm{O}^{\prime}$ Connor $\mathrm{T}$, Protzer $\mathrm{U}$, Heikenwalder $\mathrm{M}$. The direct and indirect roles of HBV in liver cancer: prospective markers for HCC screening and potential therapeutic targets. The Journal of pathology. 2015; 235: 355-67.

25. Seo YS, Jang BK, Um SH, Hwang JS, Han KH, Kim SG, et al. Validation of risk prediction models for the development of HBV-related HCC: a retrospective multi-center 10-year follow-up cohort study. Oncotarget. 2017; 8: 113213-24.

26. Ohmura Y, Takeda Y, Katsura Y, Nakahira S, Morimoto Y, Ishida T, et al. [Laparoscopic Liver Resection for HCC Recurrence]. Gan to kagaku ryoho Cancer \& chemotherapy. 2015; 42: 1884-6.

27. Li JJ, Pan K, Gu MF, Chen MS, Zhao JJ, Wang H, et al. Prognostic value of soluble MICA levels in the serum of patients with advanced hepatocellular carcinoma. Chinese journal of cancer. 2013; 32: 141-8.

28. Awan FM, Naz A, Obaid A, Ali A, Ahmad J, Anjum S, et al. Identification of Circulating Biomarker Candidates for Hepatocellular Carcinoma (HCC): An Integrated Prioritization Approach. PloS one. 2015; 10: e0138913.

29. Hu H, Gao L, Wang C, Li Y, Ma H, Chen L, et al. Lower serum soluble-EGFR is a potential biomarker for metastasis of HCC demonstrated by N-glycoproteomic analysis. Discovery medicine. 2015; 19: 333-41.

30. Fukuda MN, Nozawa S. Trophinin, tastin, and bystin: a complex mediating unique attachment between trophoblastic and endometrial epithelial cells at their respective apical cell membranes. Seminars in reproductive endocrinology. 1999; 17: 229-34.

31. Li CW, Chen BS. Investigating core genetic-and-epigenetic cell cycle networks for stemness and carcinogenic mechanisms, and cancer drug design using big database mining and genome-wide next-generation sequencing data. Cell cycle (Georgetown, Tex). 2016; 15: 2593-607.

32. Hu S, Yuan H, Li Z, Zhang J, Wu J, Chen Y, et al. Transcriptional response profiles of paired tumor-normal samples offer novel perspectives in pan-cancer analysis. Oncotarget. 2017; 8: 41334-47.

33. Lian Y, Fan W, Huang Y, Wang H, Wang J, Zhou L, et al. Downregulated Trophinin-Associated Protein Plays a Critical Role in Human Hepatocellular Carcinoma Through Upregulation of Tumor Cell Growth and Migration. Oncology research. 2018; 26: 691-701.

34. Levrero M, Zucman-Rossi J. Mechanisms of HBV-induced hepatocellular carcinoma. Journal of hepatology. 2016; 64: S84-s101.

35. Notarpaolo A, Layese R, Magistri P, Gambato M, Colledan M, Magini G, et al. Validation of the AFP model as a predictor of HCC recurrence in patients with viral hepatitis-related cirrhosis who had received a liver transplant for HCC. Journal of hepatology. 2017; 66: 552-9.

36. Kim H, Sohn A, Yeo I, Yu SJ, Yoon JH, Kim Y. Clinical Assay for AFP-L3 by Using Multiple Reaction Monitoring-Mass Spectrometry for Diagnosing Hepatocellular Carcinoma. Clinical chemistry. 2018; 64: 1230-8.

37. Abdel-Fatah TMA, Balls G, Miles AK, Moseley P, Green A, Rees R, et al. Identification of Trophinin associated protein (TROAP) as a novel biological marker in breast cancer (BC): Co-expression of TROAP and TOPO2A predicts response of anthracycline based chemotherapy (ATC-CT). Cancer Res. 2012; 72: 2.

38. Wang L, Huang J, Jiang M, Lin H, Qi L, Diao H. Activated PTHLH coupling feedback phosphoinositide to G-protein receptor signal-induced cell adhesion network in human hepatocellular carcinoma by systems-theoretic analysis. TheScientificWorldJournal. 2012; 2012: 428979

39. Chen XM, Kitts DD. Characterization of antioxidant and anti-inflammatory activities of bioactive fractions recovered from a glucose-lysine Maillard reaction model system. Molecular and cellular biochemistry. 2012; 364: 147-57. 